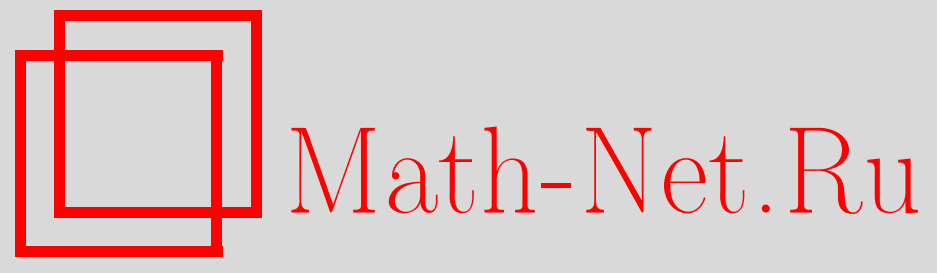

С. С. Марченков, О строении эквационально замкнутых классов, Дискрет. матем., 2006, том 18, выпуск 4, 18-30

DOI: https://doi.org/10.4213/dm70

Использование Общероссийского математического портала Math-Net.Ru подразумевает, что вы прочитали и согласны с пользовательским соглашением http://www. mathnet.ru/rus/agreement

Параметры загрузки:

IP: 54.164 .48 .24

26 апреля 2023 г., 14:24:42

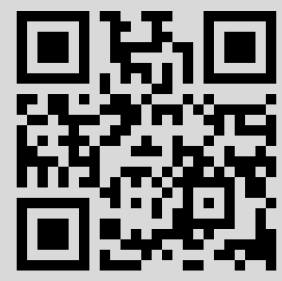




\title{
О строении эквационально замкнутых классов
}

\author{
() 2006 г. . С. Марченков
}

\begin{abstract}
Исследуется строение эквационально замкнутых классов. Доказывается теорема о представлении графика функции из эквационально замкнутого класса в виде объединения множеств значений специальных вектор-функций. При любом $k \geqslant 2$ устанавливается эквациональная порождаемость любого эквационально замкнутого класса из $P_{k}$ множеством всех его $k$-местных функций. Находятся все эквационально предполные классы в $P_{k}$ и доказывается критерий эквациональной полноты. Часть результатов переносится с эквационально замкнутых классов на позитивно замкнутые классы.

Работа выполнена при поддержке Российского фонда фундаментальных исследований, проект 06-01-00438.
\end{abstract}

\section{1. Введение}

В ряде работ $[1-9,12-21]$ на множестве функций многозначной логики определены операторы замыкания, которые являются существенно более сильными, чем традиционно рассматриваемый оператор суперпозиции. В частности, эти операторы при любом $k \geqslant 2$ порождают на множестве $P_{k}$ функций $k$-значной логики конечные (реже счетные) классификации. Набор средств, используемых при определении операторов замыкания, весьма разнообразен: применение чисто функциональных приемов, обращение к логикофункциональным языкам различных типов, использование идей из программирования и т. д.

В [11] автор предложил новый оператор замыкания - оператор эквационального замыкания, который базируется на широко представленной в математике идее вывода в исчислениях равенств. В [11] получены первые результаты, относящиеся к этому оператору. Они показали, что оператор эквационального замыкания значительно отличается от всех известных операторов замыкания, и это отличие проявляется уже на множестве булевых функций.

В настоящей статье мы продолжаем изучение оператора эквационального замыкания и порождаемых этим оператором классификаций на множествах функций многозначной логики. В теореме 1 устанавливается представление графиков функций из эквационально замкнутых классов, которое затем используется при получении ряда следствий. Теорема 2 показывает, что при любом $k \geqslant 2$ любой эквационально замкнутый класс функций из $P_{k}$ эквационально порождается множеством всех своих функций, зависящих от $k$ переменных. В качестве следствия получаем, что классификация множества функций $k$-значной логики, порождаемая оператором эквационального замыкания, конечна. В теореме 4 установлен критерий эквациональной полноты в $P_{k}$. Многие результаты, справедливые для 
оператора эквационального замыкания, оказались справедливыми и для оператора позитивного замыкания [6]. Соответствующая связь между этими операторами получена в следствии 3 из теоремы 1.

\section{2. Основные понятия}

Пусть $k \geqslant 2, E_{k}=\{0,1, \ldots, k-1\}, P_{k}-$ множество всех функций на $E_{k}$ (множество функций $k$-значной логики), $P_{k}^{(n)}$ - множество всех $n$-местных функций из $P_{k}$.

Для любого $n \geqslant 1$ и любого $i, 1 \leqslant i \leqslant n$, обозначим через $e_{i}^{n}\left(x_{1}, \ldots, x_{i}, \ldots, x_{n}\right)$ селекторную функцию, равную $x_{i}$.

Наряду с всюду определенными функциями $k$-значной логики будем рассматривать также частичные функции на $E_{k}$. Совокупность всех частичных функций на $E_{k}$ обозначим через $P_{k}^{*}$. Считаем, что $P_{k} \subset P_{k}^{*}$. Если $g \in P_{k}^{*}$, то через Dom $g$ обозначаем область определения функщии $g$.

На множестве $P_{k}$ считаем заданным оператор (операцию) суперпозиции (см. [10, 22]). Если $Q \subseteq P_{k}$, то через [ $Q$ ] обозначаем замыкание множества $Q$ относительно оператора суперпозиции. Для оператора суперпозиции предполагаем известными понятия замкнутого, полного и предполного множества (класса).

Перейдем к определению оператора эквационального замыкания [11]. Предполагаем, что каждая функция из $P_{k}$ имеет индивидуальное обозначение. Для обозначения функций из $P_{k}$ используем символ $f$ с индексами (функциональные константы). Символы $\varphi^{(n)}, \psi^{(n)}$ (возможно, с индексами) будут использоваться в качестве функциональных переменных с областью значений $P_{k}^{(n)}$.

Пусть $Q \subseteq P_{k}$. Обычным образом вводим понятие терма над $Q$. Пусть $f^{(n)}-$ функциональная константа (обозначающая функцию из $Q$ ), $\varphi^{(n)}-$ функциональная переменная, a $t_{1}, \ldots, t_{n}$ - индивидные переменные (не обязательно различные). Тогда выражения

$$
f^{(n)}\left(t_{1}, \ldots, t_{n}\right), \quad \varphi^{(n)}\left(t_{1}, \ldots, t_{n}\right)
$$

суть термы над $Q$. Если, далее, $t_{1}, \ldots, t_{n}$ - термы над $Q$ либо символы индивидных переменных, то выражения (1) также суть термы над $Q$.

Если $t_{1}, t_{2}-$ термы над $Q$, то выражение $t_{1}=t_{2}$ называем равенством над $Q$. Равенства $t_{1}=t_{2}$ и $t_{2}=t_{1}$ в дальнейшем не различаем. Частным случаем равенства $t_{1}=t_{2}$ называем любое равенство вида $t_{1}^{\prime}=t_{2}^{\prime}$, где выражения $t_{1}^{\prime}, t_{2}^{\prime}$ получаются из термов $t_{1}, t_{2}$ подстановкой вместо всех индивидных переменных некоторых значений из $E_{k}$. При этом все вхождения одной и той же индивидной переменной в термы $t_{1}, t_{2}$ замещаются одним и тем же значением из $E_{k}$.

Пусть $\Xi-$ конечная система равенств над $Q$. Последовательность $F_{1}, \ldots, F_{s}$ равенств, не содержащих индивидных переменных, называем выводом из системы равенств $\Xi$, если каждое равенство $F_{i}$ этой последовательности удовлетворяет одному из следующих условий:

(1) $F_{i}$ есть частный случай одного из равенств системы $\Xi$;

(2) для некоторого $j<i$ равенство $F_{i}$ получается из равенства $F_{j}$ заменой выражения вида $f^{(n)}\left(a_{1}, \ldots, a_{n}\right)$ значением $a$, где $a=f^{(n)}\left(a_{1}, \ldots, a_{n}\right)$;

(3) для некоторых $j, l<i$, равенство $F_{i}$ получается из равенства $F_{j}$ заменой выражения вида $\varphi^{(n)}\left(a_{1}, \ldots, a_{n}\right)$ значением $a$, при этом равенство $F_{l}$ имеет вид $a=\varphi^{(n)}\left(a_{1}, \ldots, a_{n}\right)$. 
Равенство $F$, не содержащее индивидных переменных, называем выводимым из системы равенств $\Xi$, если существует вывод из системы равенств $\Xi$, который содержит равенство $F$. Систему равенств $\Xi$ называем корректной, если из нее невозможно вывести два равенства вида

$$
a=\varphi^{(n)}\left(a_{1}, \ldots, a_{n}\right), \quad b=\varphi^{(n)}\left(a_{1}, \ldots, a_{n}\right),
$$

где $a \neq b$.

Пусть $Q \subseteq P_{k}, \Xi$ - корректная система равенств над $Q, \varphi^{(n)}-$ функциональная переменная, входящая в $\Xi$. Говорим, что функция $g\left(x_{1}, \ldots, x_{n}\right)$ из $P_{k}$ определяется системой равенств $\Xi$ над $Q$, если для любых $a_{1}, \ldots, a_{n} \in E_{k}$ из системы $\Xi$ выводимо равенство $a=\varphi^{(n)}\left(a_{1}, \ldots, a_{n}\right)$, где $a=g\left(a_{1}, \ldots, a_{n}\right)$. Множество всех функций, определяемых системой равенств $\Xi$, обозначим через $\Xi(Q)$. Положим

$$
\operatorname{Eq}[Q]=\bigcup \Xi(Q)
$$

где объединение берется по всем корректным системам равенств $\Xi$ над $Q$. Множество $\mathrm{Eq}[Q]$ называем эквациональным замыканием (или Еq-замыканием) множества $Q$. Если $Q=\mathrm{Eq}[Q]$, то множество $Q$ называется эквационально замкнутым.

В дальнейшем нам потребуется рассматривать частичные функщии $g\left(x_{1}, \ldots, x_{n}\right)$, определяемые системой равенств $\Xi$. Все приведенные определения при этом сохраняют силу, однако равенство вида $a=\varphi^{(n)}\left(a_{1}, \ldots, a_{n}\right)$ (для функции $g$ ) выводимо из $\Xi$, разумеется, только при условии $\left(a_{1}, \ldots, a_{n}\right) \in \operatorname{Dom} g$. Обозначение $\mathrm{Eq}[Q]^{*}$ используем для множества всех частичных функций, определяемых корректными системами равенств над $Q$.

Перейдем к определению оператора позитивного замыкания [6]. В качестве исходных символов языка Pos позитивной выразимости рассматриваем индивидные переменные, функциональные константы $f_{i}^{(n)}$ для обозначения всех функций из $P_{k}^{(n)}$, знак равенства $=$, логические связки $\&, \vee$ и квантор существования $\exists$. В понятие терма в языке Роs входят также индивидные переменные. Из равенств термов - элементарных формул языка Pos - образуются остальные формулы языка Pos: если $\Phi_{1}, \Phi_{2}$ - формулы языка Pos, a $x_{i}$ индивидная переменная, то

$$
\Phi_{1} \& \Phi_{2}, \quad \Phi_{1} \vee \Phi_{2}, \quad\left(\exists x_{i}\right) \Phi_{1}
$$

- также формулы языка Pos.

Всякая формула языка Pos c $m$ свободными переменными определяет некоторое $m$-местное отношение на $E_{k}$. Если $Q \subseteq P_{k}, \Phi\left(x_{1}, \ldots, x_{m}\right)$ - формула языка Pos с $m$ свободными переменными, все функциональные константы которой суть обозначения функций из $Q$, и $\Phi\left(x_{1}, \ldots, x_{m}\right)$ определяет отношение $\rho\left(x_{1}, \ldots, x_{m}\right)$ на $E_{k}$, то говорим, что формула $\Phi$ позитивно выражает отношение $\rho$ через функции множества $Q$. Отношение $\rho$ называем позитивно выразимым через функции множества $Q$, если существует формула, которая позитивно выражает отношение $\rho$ через функции множества $Q$.

Пусть $g\left(x_{1}, \ldots, x_{n}\right)$ - функция из $P_{k}$, а формула $\Phi\left(x_{1}, \ldots, x_{n}, y\right)$ позитивно выражает отношение $g\left(x_{1}, \ldots, x_{n}\right)=y$ через функции множества $Q$. В этом случае говорим, что формула $\Phi$ позитивно выражает функцию $g$ через функции множества $Q$. Совокупность всех функций, позитивно выразимых через функции множества $Q$, обозначим через $\operatorname{Pos}[Q]$. Множества вида $\operatorname{Pos}[Q]$ называем позитивно замкнутыми классами.

Пусть

$$
H=\left\{h_{1}\left(x_{1}, \ldots, x_{m}\right), \ldots, h_{l}\left(x_{1}, \ldots, x_{m}\right)\right\}
$$


- некоторое множество $m$-местных функций из $P_{k}$ и $g\left(x_{1}, \ldots, x_{n}\right) \in P_{k}$. Говорят, что функция $g$ сохраняет множество $H$, если для любых чисел $i_{1}, \ldots, i_{n} \in\{1,2, \ldots, l\}$ функция $g\left(h_{i_{1}}\left(x_{1}, \ldots, x_{m}\right), \ldots, h_{i_{n}}\left(x_{1}, \ldots, x_{m}\right)\right)$ принадлежит множеству $H$. Совокупность всех функций, сохраняющих множество $H$, обозначим через $\operatorname{Pol}(H)$. Нетрудно видеть, что множеству $\operatorname{Pol}(H)$ принадлежат все селекторные функции $e_{i}^{n}$.

Пусть $a=\left(a_{1}, \ldots, a_{n}\right)$ - набор из $E_{k}^{n}$. Обозначим через $\operatorname{ker}(a)$ следующее отношение эквивалентности на множестве $\{1,2, \ldots, n\}$ :

$$
\left\{(i, j): 1 \leqslant i, j \leqslant n, a_{i}=a_{j}\right\} .
$$

Пусть $f\left(x_{1}, \ldots, x_{n}\right) \in P_{k}$. Обозначим через $f^{a}$ функцию, которая получается из функщии $f$ следующим отождествлением переменных. Берем в каждом классе эквивалентности отношения $\operatorname{ker}(a)$ наименьший элемент и обозначаем их в порядке возрастания $i_{1}, i_{2}, \ldots, i_{l}$, $i_{1}=1$. Функщия $f^{a}\left(x_{i_{1}}, \ldots, x_{i_{l}}\right)$ получается теперь из функции $f\left(x_{1}, \ldots, x_{n}\right)$ заменой каждой переменной $x_{m}$ переменной $x_{i_{j}}$, где $\left(m, i_{j}\right) \in \operatorname{ker}(a)$.

Легко видеть, что имеет место равенство

$$
f\left(a_{1}, \ldots, a_{n}\right)=f^{a}\left(a_{i_{1}}, \ldots, a_{i_{l}}\right) .
$$

\section{3. Представление функций из эквационально замкнутых классов}

Теорема 1. Пусть $Q \subseteq P_{k} u g\left(x_{1}, \ldots, x_{n}\right) \in P_{k}^{*}$. Функиия $g$ принадлежит множеству $\mathrm{Eq}[Q]^{*}$ тогда и только тогда, когда график функиии $g$ представим в виде объединения множеств

$$
\left\{\left(h_{1}\left(x_{1}, \ldots, x_{k}\right), \ldots, h_{n}\left(x_{1}, \ldots, x_{k}\right), h\left(x_{1}, \ldots, x_{k}\right)\right): x_{1}, \ldots, x_{k} \in E_{k}\right\},
$$

где $h_{1}\left(x_{1}, \ldots, x_{k}\right), \ldots, h_{n}\left(x_{1}, \ldots, x_{k}\right)$ либо принадлежат множеству $[Q]$, либо являются селекторными функциями $e_{1}^{k}\left(x_{1}, \ldots, x_{k}\right), \ldots, e_{k}^{k}\left(x_{1}, \ldots, x_{k}\right)$, а функция $h\left(x_{1}, \ldots, x_{k}\right)$ входит в множество [Q]. При этом множества (3) подчиняются очевидному условию согласования: если в график функции $g$ входят значения вектор-функции

$$
\left(j_{1}\left(x_{1}, \ldots, x_{k}\right), \ldots, j_{n}\left(x_{1}, \ldots, x_{k}\right), j\left(x_{1}, \ldots, x_{k}\right)\right)
$$

(набор $\left(j_{1}, \ldots, j_{n}, j\right)$ может совпадать с набором $\left(h_{1}, \ldots, h_{n}, h\right)$ ) и для некоторых наборов $\left(b_{1}, \ldots, b_{k}\right),\left(c_{1}, \ldots, c_{k}\right)$ из $E_{k}^{k}$ выполняется равенство

$$
\left(h_{1}\left(b_{1}, \ldots, b_{k}\right), \ldots, h_{n}\left(b_{1}, \ldots, b_{k}\right)\right)=\left(j_{1}\left(c_{1}, \ldots, c_{k}\right), \ldots, j_{n}\left(c_{1}, \ldots, c_{k}\right)\right),
$$

то справедливо равенство

$$
h\left(b_{1}, \ldots, b_{k}\right)=j\left(c_{1}, \ldots, c_{k}\right) .
$$

Доказательство. Достаточность условий теоремы почти очевидна. В самом деле, считая для упрощения изложения функции $k_{1}, \ldots, h_{n}, h$ принадлежащими множеству $Q$, запишем условие вхождения вектор-функции $\left(h_{1}\left(x_{1}, \ldots, x_{k}\right), \ldots, h_{n}\left(x_{1}, \ldots, x_{k}\right), h\left(x_{1}, \ldots, x_{k}\right)\right)$ в график функции $g$ в форме равенства

$$
\varphi\left(h_{1}\left(x_{1}, \ldots, x_{k}\right), \ldots, h_{n}\left(x_{1}, \ldots, x_{k}\right)\right)=h\left(x_{1}, \ldots, x_{k}\right)
$$


(здесь функциональная переменная $\varphi$ отвечает функции $g$ ). Совокупность всех равенств вида (4) образует корректную (см. условие согласования в формулировке теоремы) систему равенств, эквационально определяющую функцию $g$.

Установим теперь необходимость условий теоремы. Пусть $\Xi-$ корректная система равенств над $Q$, которая эквационально определяет функцию $g$ через функции множества $Q$. Пусть далее $\varphi-n$-местная функциональная переменная, входящая в равенства $\Xi$, и для любого набора $\left(a_{1}, \ldots, a_{n}\right) \in \operatorname{Dom} g$ из системы равенств $\Xi$ выводимо равенство

$$
\varphi\left(a_{1}, \ldots, a_{n}\right)=a,
$$

где

$$
a=g\left(a_{1}, \ldots, a_{n}\right)
$$

Зафиксируем такой набор $\left(a_{1}, \ldots, a_{n}\right)$ из Dom $g$ и рассмотрим минимальный по длине вывод $F_{1}, \ldots, F_{s}$ равенства $(5)$ из системы равенств $\Xi$.

Предположим сначала, что при выводе равенства (5) применяются только пункты 1 и 2 определения вывода. Равенство $F_{1}$ может быть лишь частным случаем одного из равенств системы $\Xi$. Поскольку в выводе $F_{1}, \ldots, F_{s}$ пункт 3 определения вывода не применяется, упомянутое равенство (системы $\Xi$ ) имеет вид

$$
\varphi\left(t_{1}\left(x_{1}^{1}, \ldots, x_{m_{1}}^{1}\right), \ldots, t_{n}\left(x_{1}^{n}, \ldots, x_{m_{n}}^{n}\right)\right)=t\left(x_{1}^{n+1}, \ldots, x_{m_{n+1}}^{n+1}\right),
$$

где $t_{1}, \ldots, t_{n}$ - термы над $Q$ либо символы переменных, а $t$ - терм над $Q$. Равенство $F_{1}$ получается из равенства (6) подстановкой вместо всех переменных некоторых значений из $E_{k}$ :

$$
\varphi\left(t_{1}\left(b_{1}^{1}, \ldots, b_{m_{1}}^{1}\right), \ldots, t_{n}\left(b_{1}^{n}, \ldots, b_{m_{n}}^{n}\right)\right)=t\left(b_{1}^{n+1}, \ldots, b_{m_{n+1}}^{n+1}\right) .
$$

Ввиду минимальности вывода $F_{1}, \ldots, F_{s}$ оставшаяся часть вывода $F_{1}, \ldots, F_{s}$ состоит в последовательной замене выражений вида $f\left(b_{1}, \ldots, b_{m}\right)$ значениями $b$, где $f$ есть обозначение функщии из $Q$, встречающееся в термах $t_{1}, \ldots, t_{n}, t$, и $b=f\left(b_{1}, \ldots, b_{m}\right)$.

Понятно, что структура вывода $F_{1}, \ldots, F_{s}$ (то есть выбор тех или иных подформул равенства (7), к которым применяется пункт 2 определения вывода) не зависит от набора

$$
\left(b_{1}^{1}, \ldots, b_{m_{1}}^{1}, \ldots, b_{1}^{n}, \ldots, b_{m_{n}}^{n}, b_{1}^{n+1}, \ldots, b_{m_{n+1}}^{n+1}\right)
$$

- точно такой же вывод можно получить, если переменным

$$
x_{1}^{1}, \ldots, x_{m_{1}}^{1}, \ldots, x_{1}^{n}, \ldots, x_{m_{n}}^{n}, x_{1}^{n+1}, \ldots, x_{m_{n+1}}^{n+1}
$$

придать другие значения

$$
c_{1}^{1}, \ldots, c_{m_{1}}^{1}, \ldots, c_{1}^{n}, \ldots, c_{m_{n}}^{n}, c_{1}^{n+1}, \ldots, c_{m_{n+1}}^{n+1}
$$

(разумеется, в наборах (8), (10) при этом должно быть соблюдено необходимое условие: одинаковые переменные замещаются одинаковыми значениями из $E_{k}$ ). В результате будет получен вывод, вообще говоря, другого равенства $\varphi\left(c_{1}, \ldots, c_{n}\right)=c$. В целом все такие наборы $\left(c_{1}, \ldots, c_{n}, c\right)$ будут, очевидно, являться значением вектор-функции

$$
\left(h_{1}\left(x_{1}^{1}, \ldots, x_{m_{1}}^{1}\right), \ldots, h_{n}\left(x_{1}^{n}, \ldots, x_{m_{n}}^{n}\right), h\left(x_{1}^{n+1}, \ldots, x_{m_{n+1}}^{n+1}\right)\right),
$$


где $h_{1}, \ldots, h_{n}, h$ - либо функции, реализуемые выражениями $t_{1}, \ldots, t_{n}, t$, либо селекторные функщии (для случая, когда какое-либо из выражений $t_{1}, \ldots, t_{n}$ является символом переменной).

Чтобы сократить в вектор-функции (11) количество переменных, следует для всякого набора (8) произвести в равенстве (6) отождествление переменных в соответствии с отношением эквивалентности ker, определяемым набором (8). Можно считать, что при этом будут исппользованы переменные $x_{1}, \ldots, x_{k}$. Полученное после этого отождествления переменных равенство, вообще говоря, не является равенством системы $\Xi$. Тем не менее равенство (7) будет получаться из него некоторой подстановкой элементов из $E_{k}$ вместо переменных $x_{1}, \ldots, x_{k}$. Будет также сохранен и весь вывод $F_{1}, \ldots, F_{s}$ равенства (5). В результате мы придем к представлению (3) графика функции $g$. Что касается утверждения теоремы о согласовании вектор-функций из (3), то оно непосредственно следует из корректности системы равенств $\Xi$.

Предположим теперь, что при выводе равенства (5) используется также пункт 3 определения вывода. В соответствии с определением вывода найдем в последовательности $F_{1}, \ldots, F_{s}$ равенство $F_{i}$ вида (7), которое является частным случаем равенства (6) из системы равенств $\Xi$. Термы $t_{1}, \ldots, t_{n}, t$ в равенстве (6) уже могут содержать функциональные переменные $\psi$. Переставляя, быть может, равенство (7) в выводе $F_{1}, \ldots, F_{s}$ и пользуясь минимальностью числа $s$, будем предполагать, что все равенства $F_{i+1}, \ldots, F_{s}$ получаются из предыдущих равенств вывода $F_{1}, \ldots, F_{s}$ с помощью пунктов 2,3 определения вывода.

В соответствии с индуктивным определением терма в термах $t_{1}, \ldots, t_{n}, t$ можно выделить “последние" вхождения функциональных переменных (если процесс построения терма изобразить в виде дерева с корнем, то эти вхождения функциональных переменных будут располагаться в дереве наиболее близко к корню). Пусть в этих вхождениях участвуют переменные $\psi_{1}, \ldots, \psi_{l}$ (среди переменных $\psi_{1}, \ldots, \psi_{l}$ допускаются повторения). При выводе равенства (5) данные вхождения функциональных переменных порождают в выводе $F_{1}, \ldots, F_{s}$ равенства вида

$$
\psi_{1}\left(c_{1}^{1}, \ldots, c_{r_{1}}^{1}\right)=c^{1}, \quad \ldots, \quad \psi_{l}\left(c_{1}^{l}, \ldots, c_{r_{l}}^{l}\right)=c^{l},
$$

с помощью которых вхождения переменных $\psi_{1}, \ldots, \psi_{l}$ из равенства (7) элиминируются (здесь еще раз используется минимальность числа $s$ ). К равенствам (12) можно применить индуктивные соображения и получить указанные в формулировке теоремы представления графиков функций $g_{1}, \ldots, g_{l}$, отвечающих переменным $\psi_{1}, \ldots, \psi_{l}$.

Если в выводе $F_{1}, \ldots, F_{s}$ формулы $\psi_{1}\left(c_{1}^{1}, \ldots, c_{r_{1}}^{1}\right), \ldots, \psi_{l}\left(c_{1}^{l}, \ldots, c_{r_{l}}^{l}\right)$ заменить константами $c^{1}, \ldots, c^{l}$ (см. равенства (12)), то согласно выбору вхождений переменных $\psi_{1}, \ldots, \psi_{l}$ в термы $t_{1}, \ldots, t_{n}, t$ дальнейшее получение величин

$$
t_{1}\left(b_{1}^{1}, \ldots, b_{m_{1}}^{1}\right), \ldots, t_{n}\left(b_{1}^{n}, \ldots, b_{m_{n}}^{n}\right), t\left(b_{1}^{n+1}, \ldots, b_{m_{n+1}}^{n+1}\right)
$$

из величин

$$
b_{1}^{1}, \ldots, b_{m_{1}}^{1}, \ldots, b_{1}^{n}, \ldots, b_{m_{n}}^{n}, b_{1}^{n+1}, \ldots, b_{m_{n+1}+1}^{n+1}, c^{1}, \ldots, c^{l}
$$

будет происходить путем применения к величинам (14) подходящих функций $h_{1}, \ldots, h_{n}, h$ из множества $[Q]$ либо селекторных функций (см. первую половину доказательства теоремы). Используя это соображение, обратимся теперь к графику функции $g$.

Пусть в соответствии с условиями теоремы для каждой функции $g_{i}, 1 \leqslant i \leqslant l$, выбраны такие функции

$$
p_{1}^{i}\left(x_{1}, \ldots, x_{k}\right), \ldots, p_{r_{i}}^{i}\left(x_{1}, \ldots, x_{k}\right), p^{i}\left(x_{1}, \ldots, x_{k}\right),
$$


что вектор-функция (15) принимает значение $\left(c_{1}^{i}, \ldots, c_{r_{i}}^{i}, c^{i}\right)$. Если необходимо, то путем перестановки переменных и введения фиктивных переменных можно добиться того, что при всех $i$ вектор-функиия (15) принимают значения $\left(c_{1}^{i}, \ldots, c_{r_{i}}^{i}, c^{i}\right)$ на одном и том же наборе $(0,1, \ldots, k-1)$. Тогда в наборе (14) величины $c^{1}, \ldots, c^{l}$ можно заменить выражениями $p^{1}(0,1, \ldots, k-1), \ldots, p^{l}(0,1, \ldots, k-1)$. Следовательно, величины (13) получаются из $b_{1}^{1}, \ldots, b_{m_{1}}^{1}, \ldots, b_{1}^{n}, \ldots, b_{m_{n}}^{n}, b_{1}^{n+1}, \ldots, b_{m_{n+1}+1}^{n+1} p^{1}(0,1, \ldots, k-1), \ldots, p^{l}(0,1, \ldots, k-1)$ (выражение $p^{i}(0,1, \ldots, k-1)$ можно считать обозначением для $\left.c^{i}\right)$ применением к ним функций $h_{1}, \ldots, h_{n}, h$. Вновь, как в первой половине доказательства теоремы, производим отождествление переменных $x_{1}^{1}, \ldots, x_{m_{1}}^{1}, \ldots, x_{1}^{n}, \ldots, x_{m_{n}}^{n}, x_{1}^{n+1}, \ldots, x_{m_{n+1}}^{n+1}, x_{1}, \ldots, x_{k}$ в соответствии с отношением эквивалентности $\mathrm{ker}$, задаваемым набором $b_{1}^{1}, \ldots, b_{m_{1}}^{1}, \ldots, b_{1}^{n}$,

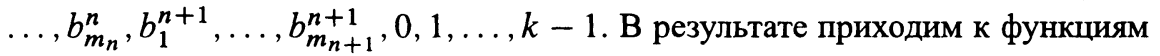

$$
h_{1}^{\prime}\left(x_{1}, \ldots, x_{k}\right), \ldots, h_{n}^{\prime}\left(x_{1}, \ldots, x_{k}\right), h^{\prime}\left(x_{1}, \ldots, x_{k}\right)
$$

из множества $[Q]$ либо селекторным функщиям, которые переводят $(0,1, \ldots, k-1)$ в набор (13). Из построения функций $h_{1}^{\prime}, \ldots, h_{n}^{\prime}, h^{\prime}$ следует также, что вектор-функция (16) будет задавать элементы графика функщии $g$ при любых значениях переменных $x_{1}, \ldots, x_{k}$. Наконец, требования теоремы о согласованности наборов значений функций (16) будут выполняться в силу корректности системы равенств $\Xi$. Теорема доказана.

Следствие 1. Пусть $Q \subseteq P_{k} u g\left(x_{1}, \ldots, x_{n}\right) \in \mathrm{Eq}[Q]^{*}$. Тогда функцию $g$ можно эквачионально определить системой равенств над $Q$, которая состоит из равенств вида

$$
\varphi\left(t_{1}\left(x_{1}, \ldots, x_{k}\right), \ldots, t_{n}\left(x_{1}, \ldots, x_{k}\right)\right)=t\left(x_{1}, \ldots, x_{k}\right),
$$

где $\varphi$ - функииональная переменная, $t_{1}, \ldots, t_{n}$ - термы над $Q$ либо символы переменных, а $t$ - терм над $Q$.

Следствие 2. Пусть $Q \subseteq P_{k}, g\left(x_{1}, \ldots, x_{n}\right) \in \mathrm{Eq}[Q]^{*}, u$ в множество $\mathrm{Eq}[Q]$ входят такие функции $f_{1}(x), \ldots, f_{k-1}(x)$, что для некоторого а $\in E_{k}$ выполняется равенство

$$
\left\{a, f_{1}(a), \ldots, f_{k-1}(a)\right\}=E_{k} .
$$

Тогда график функиии g представим в виде объединения множеств

$$
\left\{\left(j_{1}(x), \ldots, j_{n}(x), j(x)\right): x \in E_{k}\right\},
$$

где функиии $j_{1}, \ldots, j_{n}$ принадлежат множеству $\mathrm{Eq}[Q]$ либо совпадают с селекторной функций $e_{1}^{1}$, a $j \in \mathrm{Eq}[Q]$.

Доказательство. Используем представление (3) графика функции $g$. Зафиксируем вектор-функщию $\left(h_{1}, \ldots, h_{n}, h\right)$ из представления (3) и рассмотрим произвольный набор $\left(a_{1}, \ldots, a_{k}\right)$ из $E_{k}$. В соответствии с равенством (17) выберем в множестве $\left\{x, f_{1}(x), \ldots, f_{k-1}(x)\right\}$ функции $j_{1}^{\prime}(x), \ldots, j_{k}^{\prime}(x)$ так, чтобы выполнялось соотношение

$$
\left(j_{1}^{\prime}(a), \ldots, j_{k}^{\prime}(a)\right)=\left(a_{1}, \ldots, a_{k}\right) .
$$

Положим

$$
\begin{aligned}
j_{1}(x) & =h_{1}\left(j_{1}^{\prime}(x), \ldots, j_{k}^{\prime}(x)\right), \\
& \cdots \\
j_{n}(x) & =h_{n}\left(j_{1}^{\prime}(x), \ldots, j_{k}^{\prime}(x)\right), \\
j(x) & =h\left(j_{1}^{\prime}(x), \ldots, j_{k}^{\prime}(x)\right) .
\end{aligned}
$$


Очевидно, что функции $j_{1}, \ldots, j_{n}, j$ удовлетворяют условиям следствия 2 . Теперь из соотношений (19), (20) получаем, что

$$
\left(j_{1}(a), \ldots, j_{n}(a), j(a)\right)=\left(h_{1}\left(a_{1}, \ldots, a_{k}\right), \ldots, h_{n}\left(a_{1}, \ldots, a_{k}\right), h\left(a_{1}, \ldots, a_{k}\right)\right) .
$$

Следствие доказано.

Следствие 3. Пусть $Q \subseteq P_{k}$ и функция $g\left(x_{1}, \ldots, x_{n}\right) \in P_{k}$ эквачионально определяется через функции множества $Q$. Тогда g позитивно определяется через функции множества $Q$. В частности, $\mathrm{Eq}[Q] \subseteq \operatorname{Pos}[Q]$.

Доказательство. Согласно теореме 1 график функции $g$ представим в виде объединения множеств (3). Значит, отношение $y=g\left(z_{1}, \ldots, z_{n}\right)$ позитивно определяется формулой

$$
\begin{aligned}
\bigvee\left(\exists x_{1}\right) \ldots\left(\exists x_{k}\right) & \left(\left(z_{1}=h_{1}\left(x_{1}, \ldots, x_{k}\right)\right) \& \ldots\right. \\
\&\left(z_{n}\right. & \left.\left.=h_{n}\left(x_{1}, \ldots, x_{k}\right)\right) \&\left(y=h\left(x_{1}, \ldots, x_{k}\right)\right)\right)
\end{aligned}
$$

где дизъюнкция распространяется по всем наборам $\left(h_{1}, \ldots, h_{n}, h\right)$, определяющим график функции $g$. Следствие доказано.

Следствие 4. При любом $k \geqslant 2$ система всех констант $\{0,1, \ldots, k-1\}$ позитивно полна в классе $P_{k}$.

Доказательство. Согласно предложению 1 из [11]

$$
\mathrm{Eq}[\{0,1, \ldots, k-1\}]=P_{k} .
$$

Далее применяем следствие 3.

\section{4. Конечное порождение эквационально замкнутых классов и критерий эквациональной полноты}

Теорема 2. Любой эквачионально замкнутый класс функций из $P_{k}$ эквачионально порождается множеством всех своих функций, зависящих не более чем от $k$ переменных.

Доказательство. Пусть $Q$ - эквационально замкнутый класс, $Q \subseteq P_{k}$, и пусть $f\left(x_{1}, \ldots, x_{n}\right) \in Q$, где $n>k$. Рассмотрим произвольный набор $a=\left(a_{1}, \ldots, a_{n}\right)$ из $E_{k}^{n}$. Очевидно, что помимо равенства (2) функция $f$ будет также удовлетворять равенству

$$
f\left(b_{1}, \ldots, b_{n}\right)=f^{b}\left(b_{i_{1}}, \ldots, b_{i_{l}}\right)
$$

для любого набора $b=\left(b_{1}, \ldots, b_{n}\right)$, где $\operatorname{ker}(b)=\operatorname{ker}(a)$. Иными словами, если при получении функции $f^{a}$ из функции $f$ переменные $x_{1}, \ldots, x_{n}$ заменяются соответственно переменными $x_{j_{1}}, \ldots, x_{j_{n}}$, где $\left\{j_{1}, \ldots, j_{n}\right\}=\left\{i_{1}, \ldots, i_{l}\right\}$ и $j_{1}=1$, то равенство

$$
\varphi\left(x_{j_{1}}, \ldots, x_{j_{n}}\right)=f^{a}\left(x_{i_{1}}, \ldots, x_{i_{l}}\right)
$$

позволяет правильно определять значения функции $f$ на всех наборах $b$, удовлетворяющих условию $\operatorname{ker}(b)=\operatorname{ker}(a)$. Отсюда сразу следует, что система равенств вида (21), рассматриваемая для всех наборов $a$ из $E_{k}^{n}$, корректно определяет функцию $f$ через функции множеств $Q^{(l)}, l \leqslant k$. Теорема доказана. 
Следствие 5. При любом $k \geqslant 2$ число эквачионально замкнутых классов в $P_{k}$ конечно.

Следствие 6. При любом $k \geqslant 2$ любой позитивно замкнутый класс функций из $P_{k}$ позитивно порождается множеством всех свочх функций, зависящих не более чем от $k$ переменных.

Доказательство. Согласно следствию 3 из теоремы 1, любой позитивно замкнутый класс состоит из нескольких эквационально замкнутых классов.

Назовем множество функщий $Q \subseteq P_{k}$ тотальным, если для любого $a \in E_{k}$ в множество $Q$ входит функция, принимающая значение $a$.

Нетрудно видеть, что для любого тотального множества $Q \subseteq P_{k}$, замкнутого относительно операции отождествления переменных, множество всех функций из $Q$, зависящих не более чем от $k$ переменных, также является тотальным.

Следующая теорема усиливает предложение 4 из [11].

Теорема 3. Пусть $Q$ - тотальное множество функиий из $P_{k}$, замкнутое относительно операчий отождествления и перестановки переменных, а также введения фиктивных переменных. Тогда

$$
\operatorname{Pol}\left(Q^{(k)}\right) \subseteq \operatorname{Eq}[Q]
$$

Доказательство. Пусть $g\left(x_{1}, \ldots, x_{n}\right)$ - произвольная функция, лежащая в $\operatorname{Pol}\left(Q^{(k)}\right)$, и $\left(a_{1}, \ldots, a_{n}\right) \in E_{k}^{n}$. Ввиду тотальности множества $Q$ в нем найдутся такие функции $f_{1}, \ldots, f_{n}$, которые принимают соответственно значения $a_{1}, \ldots, a_{n}$. Путем отождествления переменных образуем из этих функций функции $f_{1}^{\prime}, \ldots, f_{n}^{\prime}$, которые принадлежат множеству $Q$, зависят не более чем от $k$ переменных и также принимают значения $a_{1}, \ldots, a_{n}$. Можно считать, что наборы, на которых функщии $f_{1}^{\prime}, \ldots, f_{n}^{\prime}$ принимают значения $a_{1}, \ldots, a_{n}$, не содержат одинаковых компонент. С помощью введения фиктивных переменных получаем далее из функций $f_{1}^{\prime}, \ldots, f_{n}^{\prime}$ функции $g_{1}, \ldots, g_{n}$, которые принадлежат множеству $Q^{(k)}$ и принимают значения $a_{1}, \ldots, a_{n}$ на наборах, не имеющих одинаковых компонент. Наконец, переставляя, быть может, в функциях $g_{1}, \ldots, g_{n}$ переменные, добиваемся того, что полученные после перестановки переменных функщии $g_{1}^{\prime}, \ldots, g_{n}^{\prime}$ принимают значения $a_{1}, \ldots, a_{n}$ на одном и том же наборе $(0,1, \ldots, k-1)$. Итак,

$$
\left(g_{1}^{\prime}(0,1, \ldots, k-1), \ldots, g_{n}^{\prime}(0,1, \ldots, k-1)\right)=\left(a_{1}, \ldots, a_{n}\right) .
$$

Если теперь

$$
h\left(x_{1}, \ldots, x_{k}\right)=g\left(g_{1}^{\prime}\left(x_{1}, \ldots, x_{k}\right), \ldots, g_{n}^{\prime}\left(x_{1}, \ldots, x_{k}\right)\right),
$$

где функция $h$ ввиду включения $g \in \operatorname{Pol}\left(Q^{(k)}\right)$ принадлежит множеству $Q^{(k)}$, то рассматриваем равенство

$$
\varphi\left(g_{1}^{\prime}\left(x_{1}, \ldots, x_{k}\right), \ldots, g_{n}^{\prime}\left(x_{1}, \ldots, x_{k}\right)\right)=h\left(x_{1}, \ldots, x_{k}\right)
$$

над множеством $Q$. Совокупность всех таких равенств (по всем наборам $\left(a_{1}, \ldots, a_{n}\right)$ из $\left.E_{k}^{n}\right)$ и будет, очевидно, эквационально определять функцию $g$ через функции множества $Q$. Теорема доказана.

Следствие 7. Пусть $Q-$ тотальный эквачионально замкнутый класс функций из $P_{k}$. Тогда

$$
Q=\operatorname{Pol}\left(Q^{(k)}\right)
$$


Доказательство. Включение $\operatorname{Pol}\left(Q^{(k)}\right) \subseteq Q$ установлено в теореме 3. Обратное включение следует из замкнутости класса $Q$ относительно операции суперпозиции (см. [11]).

Следствие 8. В условиях теоремы 3 имеет место включение

$$
\operatorname{Pol}\left(Q^{(k)}\right) \subseteq \operatorname{Pos}[Q]
$$

Для доказательства достаточно применить следствие 3 из теоремы 1.

Следствие 9. Пусть $Q-$ позитивно замкнутый тотальный класс функций из $P_{k}$. Тогда

$$
Q=\operatorname{Pol}\left(Q^{(k)}\right) \text {. }
$$

Доказательство. Включение $Q \subseteq \operatorname{Pol}\left(Q^{(k)}\right)$ вытекает из того, что функции класса $Q$ (замкнутого, в частности, относительно операции суперпозиции) сохраняют множество $Q^{(k)}$. Далее применяем следствие 5 .

Следствие 10. Пусть $Q-$ тотальное множество, $Q \subseteq P_{k}$. Тогда существует не более одного позитивно замкнутого класса $R$ из $P_{k}$, для которого $R^{(k)}=Q^{(k)}$.

Введение дополнительных ограничений на класс $Q$ приводит к следующему утверждению.

Предложение 1. Пусть $Q$ - эквачионально замкнутый класс, $Q \subseteq P_{k}$ и существуют такой элемент $a \in E_{k}$ и такие функиии $f_{1}, \ldots, f_{k-1} \in Q^{(1)}$, что выполняется равенство (17). Тогда

$$
Q=\operatorname{Eq}\left[Q^{(1)}\right] \text {. }
$$

Доказательство. В самом деле, из условия (17) следует, что значением вектор-функции

$$
\left(x, f_{1}(x), \ldots, f_{k-1}(x)\right)
$$

в точке $x=a$ служит набор из $E_{k}^{k}$, не имеющий одинаковых компонент. Если $f\left(x_{1}, \ldots, x_{n}\right)$ - произвольная функция из $Q$, то ввиду замкнутости $Q$ относительно операции суперпозиции при подстановке в функцию $f$ на места всех переменных любых функций из набора (22) получается некоторая функция из $Q^{(1)}$. Отсюда и из свойства вектор-функции (22) вытекает, что функцию $f$ можно эквационально определить через функции множества $Q^{(1)}$ системой равенств, состоящей, например, из всех равенств вида

$$
\varphi\left(g_{1}(x), \ldots, g_{n}(x)\right)=g(x),
$$

где $g_{1}(x), \ldots, g_{n}(x) \in\left\{x, f_{1}(x), \ldots, f_{k-1}(x)\right\}$ и

$$
g(x)=f\left(g_{1}(x), \ldots, g_{n}(x)\right) .
$$

Предложение доказано.

Пусть $D$ - непустое собственное подмножество множества $E_{k}, \pi$ - перестановка на множестве $E_{k}$, которая разлагается в произведение циклов одной и той же простой длины. Обозначим через $Q_{D}$ множество всех функций из $P_{k}$, которые сохраняют множество $D$, и через $Q_{\pi}-$ множество всех функций $f \in P_{k}$, самодвойственных относительно перестановки $\pi$, то есть удовлетворяющих тождеству

$$
\pi\left(f\left(x_{1}, \ldots, x_{n}\right)\right)=f\left(\pi\left(x_{1}\right), \ldots, \pi\left(x_{n}\right)\right) .
$$


Пусть $\mathbf{T}_{k}$ есть множество функщий $P_{k}^{(1)}$, рассматриваемое как полугруппа с операцией композиции (суперпозиции). Полугруппа $T \subseteq \mathbf{T}_{k}$ называется транзитивной, если для любых двух элементов $a, b \in E_{k}$ в $T$ существует функция $f$, для которой $f(a)=b$.

Лемма 1. Пусть $T$ - максимальная (по включению) из транзитивных подполугрупп полугруппы $\mathbf{T}_{k}$, которая не содержится в классах типа $Q_{\pi}$ и обладает следующим свойством: найдутся такие неравные элементы $a_{1}, a_{2} \in E_{k}$, что для любых двух функиий $f_{1}, f_{2} \in T$ либо вектор-функиия

$$
\left(f_{1}(x), f_{2}(x)\right)
$$

не принимает значение $\left(a_{1}, a_{2}\right)$, либо существует элемент $b \in E_{k}$, для которого $f_{1}(b)=f_{2}(b)$.

Тогда класс $\operatorname{Pol}(T)$ Eq-предполон в $P_{k}$.

Доказательство. Покажем, что $\mathrm{Eq}[T] \neq P_{k}$. В самом деле, если $g(x) \in \mathrm{Eq}[T]$, то по теореме 1 график функции $g$ представим в виде объединения множеств значений вектор-функций (23), где $f_{1}, f_{2} \in T$. Однако по свойству полугруппы $T$ либо для некоторых неравных $a_{1}, a_{2}$ любая вектор-функция (23) не принимает значение $\left(a_{1}, a_{2}\right)$ (и тогда $\left.g\left(a_{1}\right) \neq a_{2}\right)$, либо существуют элемент $b$ и вектор-функция (23), для которой $f_{1}(b)=f_{2}(b)$ (и тогда соответственно $g(b)=b$ ). Таким образом, функция $g$ либо удовлетворяет неравенству $g\left(a_{1}\right) \neq a_{2}$, либо имеет неподвижную точку $b$. Понятно, что в множестве $P_{k}^{(1)}$ содержатся функции, переводящие $a_{1}$ в $a_{2}$ и не имеющие неподвижных точек. Следовательно, $\mathrm{Eq}[T] \neq P_{k}$.

Далее, согласно теореме 3 класс $\mathrm{Eq}[T]$ содержит множество $\operatorname{Pol}(T)$. Возьмем произвольную функщию $h\left(x_{1}, \ldots, x_{n}\right)$, не принадлежащую множеству $\operatorname{Pol}(T)$, и покажем, что множество $T \cup\{h\}$ Еq-полно в $P_{k}$. Так как $\mathrm{Eq}[T] \neq P_{k}$, из этого будет следовать, что $\mathrm{Eq}[T]=\operatorname{Pol}(T)$ и $\operatorname{Pol}(T)$ является Eq-предполным классом.

Из соотношения $h \notin \operatorname{Pol}(T)$ вытекает, что в полугруппе $T$ найдутся такие функции $f_{1}, \ldots, f_{n}$, что функция

$$
f(x)=h\left(f_{1}(x), \ldots, f_{n}(x)\right),
$$

принадлежащая замыканию $[T \cup\{h\}]$, не входит в полугруппу $T$. Ввиду свойства максимальности полугруппы $T$ (транзитивная полугруппа) $T^{\prime}=[T \cup\{h\}]$ будет удовлетворять следующему условию: для любых двух неравных элементов $a_{1}, a_{2}$ из $E_{k}$ в $T^{\prime}$ найдутся такие функции $f_{1}, f_{2}$, что для некоторого $c \in E_{k}$ выполняются соотношения

$$
f_{1}(c)=a_{1}, \quad f_{2}(c)=a_{2}, \quad(\forall x)\left(f_{1}(x) \neq f_{2}(x)\right) .
$$

В этом случае согласно теореме 3 из [11] множество $T^{\prime}$ будет Еq-полным в $P_{k}$, если только оно целиком не содержится ни в одном из Eq-предполных классов типов $Q_{D}$ и $Q_{\pi}$. Соотношение $Q \nsubseteq Q_{D}$ сразу следует из транзитивности полугруппы $T^{\prime}$, а соотношение $Q \nsubseteq Q_{\pi}-$ из соответствующего соотношения $T \nsubseteq Q_{\pi}$, которое имеется в условиях леммы. Лемма доказана.

Теорема 4. Система функций из $P_{k}$ является Еq-полной в классе $P_{k}$ тогда и только тогда, когда она целиком не содержится ни в одном из следуюших Еq-замкнутых классов:

(1) предполные (относительно операчии суперпозищии) в $P_{k}$ классы типа $Q_{D}$;

(2) предполные (относительно операчии суперпозичии) в $P_{k}$ классы типа $Q_{\pi}$; 
(3) Еq-предполные классы вида $\operatorname{Pol}(T)$, где $T$ - подполугруппа полугруппы $\mathbf{T}_{k}$, удовлетворяющая условиям леммы 1.

Доказательство. Необходимость условий теоремы следует из Еq-неполноты классов типа $Q_{D}$ и $Q_{\pi}$, доказанной в [11] (следствие 1 из предложения 2 и предложение 3 ), и из Eq-неполноты классов типа $\operatorname{Pol}(T)$, установленной в лемме 1.

Докажем достаточность условий теоремы. Пусть система функций $Q \subseteq P_{k}$ целиком не содержится ни в одном из Еq-замкнутых классов пунктов 1-3 теоремы. Будем предполагать, что $Q$ является Еq-замкнутым классом.

Согласно предложению 5 в [11] невхождение системы $Q$ в классы 1,2 обеспечивает транзитивность полугруппы $T=(\mathrm{Eq}[Q])^{(1)}$. Согласно доказанному выше предложению 1

$$
Q=\operatorname{Eq}[Q]=\operatorname{Pol}(T) \text {. }
$$

Если $T$ не обладает свойством, указанным в лемме 1 , то по теореме 3 из [11] система $Q$ является Еq-полной в $P_{k}$.

Пусть для некоторого Eq-предполного класса $Q_{\pi}$ выполняется включение $T \subseteq Q_{\pi}$. Тогда множество $Q_{\pi}^{(1)}$ также является транзитивной полугруппой. Значит, согласно предложению 1

$$
Q_{\pi}=\operatorname{Eq}\left[Q_{\pi}^{(1)}\right] .
$$

Из включения $T \subseteq Q_{\pi}^{(1)}$ следует, что

$$
\mathrm{Eq}[T] \subseteq Q_{\pi}
$$

Сравнивая теперь (24), (25) и используя очевидное включение $\operatorname{Pol}(T) \subseteq \operatorname{Eq}[T]$, приходим к соотношению $\mathrm{Eq}[Q] \subseteq Q_{\pi}$, что противоречит условию теоремы о невхождении системы $Q$ в классы типа $Q_{\pi}$.

Итак, далее можно считать, что полугруппа $T$ целиком не входит ни в один из Eq-предполных классов $Q_{\pi}$. Предположим, что $T$ обладает указанным в лемме 1 свойством с неравными элементами $a_{1}, a_{2}$. Ввиду следствия 2 из теоремы 1 график произвольной функции $g\left(x_{1}, \ldots, x_{n}\right) \in Q$ представим в виде объединения множеств (18), где функции $j_{1}, \ldots, j_{n}$ либо входят в $T$, либо совпадают с функцией $e_{1}^{1}$, а $j-$ функция из $T$. Рассмотрим (для данных $a_{1}, a_{2}$ ) максимальную по включению подполугруппу $T^{\prime}$ полугруппы $\mathbf{T}_{k}$, которая содержит полугруппу $T$ и обладает свойством, указанным в лемме 1 . Тогда в силу включения $T \subseteq T^{\prime}$ и следствия 2 из теоремы 1 будет также справедливым включение $\mathrm{Eq}[T] \subseteq \mathrm{Eq}\left[T^{\prime}\right]$. Из равенства (24) и определения операторов $\mathrm{Eq}$, Роl получаем, что $\mathrm{Eq}[T]=Q$. Таким образом, $Q \subseteq \mathrm{Eq}\left[T^{\prime}\right]$, что противоречит условию теоремы о невхождении системы $Q$ в Еq-предполные классы пункта 3. Теорема доказана.

\section{Список литературы}

1. Голунков Ю. В., Полнота систем функций в операторных алгоритмах, реализующих функции $k$-значной логики. Вероятностные методы и кибернетика (1980) №17, 23-34.

2. Данильченко А. Ф., О параметрической выразимости функций трехзначной логики. Алгебра $и$ логика (1977) 16, №4, 397-416.

3. Кузнецов А. В., О средствах для обнаружения невыводимости и невыразимости. В кн.: Логический вывод. Наука, Москва, 1979, с. 5-33.

4. Марченков С. С., $S$-классификация функций многозначной логики. Дискретиая математика (1997) 9, №3, 125-152. 
5. Марченков С. С., $A$-классификация функций многозначной логики. Докл. $P A H$ (1999) 366, №4, 455-457.

6. Марченков С. С., О выразимости функций многозначной логики в некоторых логикофункциональных языках. Дискретная математика (1999) 11, №4, 110-126.

7. Марченков С. С., Замкиутые классы булевых фуикций. Физматлит, Москва, 2000.

8. Марченков C. С., S-классификачия функиий трехзначной логики. Физматлит, Москва, 2001.

9. Марченков С. С., Операторы замыкания с разветвлением по предикату. Вестник МГУ. Серия 1. Математика. Механика. (2003) №6, 37-39.

10. Марченков С. С., Фуикциональные системы с операцией суперпозиции. Физматлит, Москва, 2004.

11. Марченков С. С., Эквациональное замыкание. Дискретиая математика (2005) 17, №2, 117-126.

12. Нгуен Ван Хоа, Об $L$-эквивалентности систем функций в многозначных логиках. Алгебра $u$ логика (1988) 27, №1, 37-47.

13. Нгуен Ван Хоа, О $G$-полных замкнутых классах $k$-значной логики. EIK (1990) 26, №5/6, 301313.

14. Нгуен Ван Хоа, К описанию семейства $G$-полных замкнутых классов $k$-значной логики. Кибернетика (1990) 5, 9-12.

15. Нгуен Ван Хоа, О структуре самодвойственных замкнутых классов трехзначной логики $P_{3}$. Дискретная математика (1992) 4, №4, 82-95.

16. Нгуен Ван Хоа, О семействах замкнутых классов $k$-значной логики, сохраняемых всеми автоморфизмами. Дискретиая мателатика (1993) 5, №4, 87-108.

17. Нгуен Ван Хоа, О замкнутых класса $k$-значной логики, самодвойственных относительно транзитивных групп. Дискретная математика (1998) 8, №1, 129-156.

18. Соловьев В. Д., Замкнутые классы в $k$-значной логике с операцией разветвления по предикату. Дискретная математика (1990) 2, №4, 19-25.

19. Тайманов В. А., О функциональных системах $k$-значной логики с операциями программного типа. Докл. АН СССР (1983) 268, №6, 1307-1310.

20. Тарасова О. С., Классы $k$-значной логики, замкнутые относительно расширенной операции суперпозиции. Вестиик МГУ. Серия 1. Математика. Механика (2001) №6, 54-57.

21. Тарасова О. С., Классы функций трехзначной логики, замкнутые относительно операции суперпозиции и перестановки. Вестиик МГУ. Серия 1. Математика. Механика (2004) №1, 25-29.

22. Яблонский С. В., Введение в дискретиую математику. Наука, Москва, 1986.

Статья поступила 3.11.2005. 